\title{
The Stratification Analysis of Sediment Data for Lake Michigan
}

\author{
Xiangsheng Xia ${ }^{1}$ and David H. Miller ${ }^{2}$ \\ ${ }^{1}$ CSC Corporation and ${ }^{2}$ US EPA
}

\begin{abstract}
Accurately understanding the distribution of sediment measurements within large water bodies such as Lake Michigan is critical for modeling and understanding of carbon, nitrogen, silica, and phosphorus dynamics. Several water quality models have been formulated and applied to the Great Lakes to investigate the fate and transport of nutrients and other constituents, as well as plankton dynamics.

This paper summarizes the development of spatial statistical tools to study and assess the spatial trends of the sediment data sets, which were collected from Lake Michigan, as part of Lake Michigan Mass Balance Study. Several new spatial measurements were developed to quantify the spatial variation and continuity of sediment data sets under concern. The applications of the newly designed spatial measurements on the sediment data, in conjunction with descriptive statistics, clearly reveal the existence of the intrinsic structure of strata, which is hypothesized based on linear wave theory. Furthermore, a new concept of strata consisting of two components defined based on depth is proposed and justified. The findings presented in this paper may impact the future studies of sediment within Lake Michigan and all of the Great Lakes as well.
\end{abstract}

Key words: Spatial statistics, stratification, sediment nutrients and carbon, Lake Michigan Mass Balance Study.

\section{Introduction}

The focus of this research is the data sets of sediment measurements in Lake Michigan for nutrients which include data for phosphorus (total phosphorus, sodium hydroxide extractable phosphorus), biogenic silica, total organic nitrogen, and organic carbon. All data sets were collected as part of the Lake Michigan Mass Balance Study, which was conducted by the United States Environmental Protection Agency for the period of 1994 - 1996. Details of sample analysis and sampling techniques can be found in the Lake Michigan Mass Balance Project Methods Compendium (U.S. Environmental Protection Agency, 1997). 
In this research, we utilize data collected at 116 stations in Lake Michigan, which were widely dispersed throughout the lake. The sediment samples were collected using box cores, gravity cores, and ponars and the data utilized in the present research represents the surficial sediment, the top $1 \mathrm{~cm}$ of the sediment. This data comprises the most extensive collection of samples in the lake in the past two decades. It provides a thorough coverage of the lake bed appropriate for application of geospatial methods.

The preliminary data analysis, such as histograms and contour plots of the distributions of sediment parameters, suggests strong correlation between the distribution of sediment parameters and the water depth where the samples were collected. Furthermore, the distribution of sediment nutrient and carbon measurements within Lake Michigan is observed to vary considerably from one area to another. The samples collected from near-shore are collectively lower than the samples from offshore, while the samples from the center of the lake are significant higher than that of the rest of the lake. This phenomenon poses a serious problem for accurately predicting the grid points at unsampled locations. The challenge of data estimation when samples are not homogeneous across the area is how the characteristics of underlying natural phenomena represented by the samples, such as means, trends, and continuity can be preserved. All estimation methods, including inverse distance square, natural neighbor, or Kriging, are similar in that estimations are calculated by linearly combining weighted samples. The difference is in how the weights are calculated. When grid points are adjacent to areas with samples of large variation, the estimations tend to be smoothed by potentially combining samples of significant difference, thus the original trends, variation, and continuity are not preserved. A better approach is to use samples of similar nature to compute the estimations, rather than to mix samples of different magnitude. In practice, data can be better utilized if it can be divided into groups, such that the elements of each group have a high degree of association while the groups are clearly distinct from each other.

Based on observations of the sediment data, it has been realized that sediment strata are relevant to the water depth of interaction with the hydrodynamics of the lake system. Wind energies result in dissipation of wave energy that impacts resuspension and settling of nutrients and carbon at depths corresponding to the magnitude of the wave energy. The water depth of interaction with the lake bottom can be estimated by Airy theory (proposed by George Biddell Airy in 1841), and also commonly referred to as Linear Wave Theory (Craik , 2004). This is a core theory of ocean surface waves used in ocean and coastal engineering. Both the shape and speed of a wave are resultant of the displacement of water particles. In deep water, the shape of the wave is a sine wave. As the wave approaches shore the wave motion is affected by bottom friction at a water depth 
equal to one-half the wavelength. Thus, the potential for wave and sediment interaction exists. The equation for estimating depth reached by a wave is

$$
D=\left(g T^{2} / 2 \pi\right) / 2,
$$

where the $\mathrm{D}$ is the depth $(\mathrm{m})$, of the water column at which a wave of a given wave length begins to interact with the sediment, $g$ is acceleration due to gravity $\left(\mathrm{m} / \mathrm{s}^{2}\right)$, and $\mathrm{T}$ is wave period (s) (Rosemann, and Seibel (1977), Dean, and Dalrymple (1991), and Craik (2004)). The distribution of water depth of interaction with the lake bed was calculated from equation (1) using data acquired from two buoys in the lake, (Miller, Xia, Huang, and Rossmann (2010)). A total of 120,743 observations from buoy number 45002 maintained in the northern basin of the lake and 123,704 observations from buoy number 45007 maintained in the southern basin were used in conjunction with equation (1) to calculate depth of first interaction with the sediment for each observed wave. In examining the calculated depths, the value of $40 \mathrm{~m}$ represents approximately the 98th percentile for each buoy. There were no depths at which a wave of a given wavelength begins interaction with the sediment greater than $100 \mathrm{~m}$. Thus based on the frequency of water column depth at which a wave of a given wavelength begins interaction with the sediment, strata were estimated as non-depositional (0 - 40 $\mathrm{m})$, transitional $(40-100 \mathrm{~m})$, and depositional $(>100 \mathrm{~m})$.

To vindicate the definition of strata, a set of descriptive statistics including group means and standard deviations of the means were calculated for sediment samples on each stratum for all given variables. Examination of descriptive statistics indicates that strata classification based on linear wave theory is reasonably good since the summaries of each individual stratum are significantly different from the others. For example, as indicated in Table 1, the mean of total phosphorus samples from the non-depositional zone is 0.201 while the mean of samples from the transitional zone is $0.547,272 \%$ higher than that from the shallow zone. Furthermore, the mean of TP from the depositional zone is 1.255 , which is $229 \%$ higher than that of the transitional zone. A similar phenomenon was observed for all parameters of the sediment study (Table 1).

Mean, median, standard deviation, quartiles, and other traditional descriptive statistics summaries are not very effective to describe the distribution and variation patterns of data since every datum in a data set is treated as an independent random observation, and this appproach fails to take account the spatial features associated with data sets. Spatial relationships such as spatial continuity exist in most environmental study data. Furthermore, the information regarding the locations of extreme observations, the overall trend, the degree of continuity of regionalized variables, the direction and gradient, among others, are crucial for the application of data interpolation, extrapolation, and strata recognition. It 
Table 1: Summary of select descriptive statistics calculated using sediment data

\begin{tabular}{lccc}
\hline \multicolumn{4}{c}{ Total Phosphorus (Unit: } \\
Conf. of Strata & Data Range & Mean & Std. Deviation \\
\hline Non-Depositional Zone & $0.029-0.623$ & 0.201 & 0.134 \\
Transitional Zone & $0.096-1.215$ & 0.547 & 0.413 \\
Depositional Zone & $0.435-1.447$ & 1.255 & 0.182 \\
All Samples & $0.029-1.447$ & 0.753 & 0.514 \\
\hline Sodium Hydroxide Extractable Phosphorus (Unit: mg/g dry wt.) \\
Conf. of Strata & Data Range & Mean & Std. Deviation \\
\hline Non-Depositional Zone & $0.007-0.147$ & 0.033 & 0.029 \\
Transitional Zone & $0.006-0.247$ & 0.076 & 0.055 \\
Depositional Zone & $0.062-0.285$ & 0.193 & 0.059 \\
All Samples & $0.006-0.285$ & 0.113 & 0.085 \\
\hline
\end{tabular}

\begin{tabular}{lccc}
\hline \multicolumn{4}{c}{ Organic Carbon (Unit: } \\
Conf. of Strata & Data Range & Mean & Std. Deviation \\
\hline Non-Depositional Zone & $0.400-21.20$ & 3.436 & 4.396 \\
Transitional Zone & $0.500-48.51$ & 12.763 & 12.532 \\
Depositional Zone & $10.28-47.875$ & 33.68 & 6.802 \\
All Samples & $0.400-48.51$ & 18.868 & 15.353 \\
\hline
\end{tabular}

\begin{tabular}{lccc}
\hline \multicolumn{4}{c}{ Total Organic Nitrogen (Unit: $\mathbf{m g}$ /g dry wt.) } \\
Conf. of Strata & Data Range & Mean & Std. Deviation \\
\hline Non-Depositional Zone & $0.100-3.200$ & 0.588 & 0.683 \\
Transitional Zone & $0.100-7.200$ & 1.918 & 1.775 \\
Depositional Zone & $2.400-7.050$ & 5.041 & 0.964 \\
All Samples & $0.100-7.200$ & 2.812 & 2.241 \\
\hline \multicolumn{5}{c}{ Biogenic Silica (Unit: mg/g dry wt.) } \\
Conf. of Strata & Data Range & Mean & Std. Deviation \\
\hline Non-Depositional Zone & $0.313-34.110$ & 4.520 & 7.398 \\
Transitional Zone & $0.182-40.441$ & 10.869 & 9.975 \\
Depositional Zone & $17.622-105.792$ & 51.434 & 26.338 \\
All Samples & $0.182-105.792$ & 25.316 & 27.589 \\
\hline
\end{tabular}

is the localized information of samples, rather than the global statistics of data sets, that provides the most important key elements for accurately predicting the estimation for grid points that are in close vicinity to the available observations. Being able to accurately provide estimations for areas of miniature scale has been proven to be critical for the practice of environmental modeling, especially for models running on high resolution grids since these models require estimation of values on a much smaller spatial scale. 
We would therefore prefer to develop some tools which can incorporate the spatially dependent information into our consideration for a better statistical inference. Specifically, we would like to measure the degree of continuity of stratified and unstratified sample sets in order to understand whether and how the classification of strata changes the perspective of the spatial continuity of the data under concern. In this research, we utilized the spatial continuity tools to develop several new statistical summaries, along with descriptive methods, to justify the creation of strata based on linear wave theory.

\section{Spatial Continuity Methods}

Often, the natural phenomenon under study can be written as a function $y=f(\mathbf{Z})$, where $\mathbf{Z}$ is a vector of two-dimensional or three-dimensional location such as $\mathbf{Z}=$ (Longitude, Latitude, Depth). We define the distance between two location vectors, $\mathbf{u}, \mathbf{v}, \operatorname{dis}(\mathbf{u}, \mathbf{v})$, and the difference between two measurements $s, t$, dif $(s, t)$, as metric functions. The distance function between two location vectors will be the conventional two or three dimensional Euclidean distance or any statistics distance (which satisfies as a metric function). We say that $y=f(\mathbf{Z})$ is continuous at $\mathbf{Z}=\mathbf{Z}_{0}$ if the difference $\operatorname{dif}\left(f(\mathbf{Z}), f\left(\mathbf{Z}_{0}\right)\right)=\left|f(\mathbf{Z})-f\left(\mathbf{Z}_{0}\right)\right|$ can be arbitrarily small as long as $\mathbf{Z}$ and $\mathbf{Z}_{0}$ are close enough.

On the other hand, the continuity of spatial samples is a concept to measure the correlation between the variation of observations and the distances at which the observations are separated. Let $\mathbf{S}=\left\{s_{1}, s_{2}, \cdots, s_{n}\right\}$ be a data set, where each data element (the measurement) of $s_{i} \in \mathbf{S}$ is collected at the location $\mathbf{v}\left(s_{i}\right)$. We will use $\operatorname{dis}\left(s_{i}, s_{j}\right)$ as a shorthand notation for $\operatorname{dis}\left(\mathbf{v}\left(s_{i}\right), \mathbf{v}\left(s_{j}\right)\right)$ in this paper. In general, we consider that the data set $\mathbf{S}$ is spatially continuous if it is most likely that $\operatorname{dif}\left(s_{i}, s_{j}\right) \leq \operatorname{dif}\left(s_{m}, s_{n}\right)$ if $\operatorname{dis}\left(s_{i}, s_{j}\right) \leq \operatorname{dis}\left(s_{m}, s_{n}\right)$. The spatial continuity therefore can be understood as a tendency, or more accurately, the probability that "data close to each other are more likely similar than data that are far apart", (Isaaks, and Srivastava (1989)).

Spatial continuity exists in most environmental data. One of the popular graphical tools for studying spatial continuity is the $h$-scatterplot, which is a plot on the $\mathbf{x y}$-plane of all possible pairs of sample values whose locations are separated by a given distance $\mathbf{h}$ in a specific direction. In some literature, the distance $\mathbf{h}$ which is a vector of a distance and a direction is referred to as lag. The $\mathbf{x}-$ and $\mathbf{y}$-coordinates of any point on an $h$-scatterplot represent the values of a pair of data whose lag is $\mathbf{h}$. Some characteristics of data sets such as abnormalities and spatial continuity are easily assessed by inspecting the associated $h$-scatterplots. If the values of a pair of samples separated by lag $\mathbf{h}$ are similar, then the point whose coordinates are the values of the pair will be plotted close to $\mathbf{y}-\mathbf{x}=\mathbf{0}$, the diagonal line passing the origin on the $\mathbf{x y}$-plane. The smaller the differences 
between paired samples with separation $\mathbf{h}$, the closer the points will be to the diagonal line. The shapes of the points on $h$-scatterplots can be used to study the trends of spatial continuity of the data sets under concern.

One of the quantitative measurements of spatial continuity is the average of differences of all samples over successive lags. As mentioned before, the difference between paired samples with lag $\mathbf{h}$ is a metric function. One such metric could be used in $h$-scatterplots is the perpendicular distance from any point to the $\mathbf{y}-\mathbf{x}=\mathbf{0}$ line, which is the shortest distance from a point to the line of $\mathbf{y}-\mathbf{x}=\mathbf{0}$. It is easy to show this perpendicular distance is

$$
d=\operatorname{dif}\left(s_{x}, s_{y}\right)=\frac{\sqrt{2}\left|s_{x}-s_{y}\right|}{2}
$$

where $s_{x}, s_{y}$ are measurements of two samples whose separation is $\mathbf{h}$. Consider the points in Figure $1(\mathrm{a}), A=\left(x_{o}, y_{o}\right), B=\left(x_{o}, x_{o}\right)$, and $Q$, where $Q$ is the point on the $\mathbf{y}=\mathbf{x}$ line and the segment $\overline{\mathbf{A Q}}$ is perpendicular to the $\mathbf{y}=\mathbf{x}$ line. Apparently, $\triangle A B Q$ is a right isosceles triangle so

$$
d=|\overline{A Q}|=|\overline{B A}| \cos \left(\frac{\pi}{4}\right)=\frac{\sqrt{2}\left|x_{o}-y_{o}\right|}{2} .
$$

The average of differences for a given distance $\mathbf{h}$ now can be described as

$$
\rho(\mathbf{h})=\frac{1}{N(\mathbf{h})} \sum_{\operatorname{dis}\left(s_{x}, s_{y}\right)=\|\mathbf{h}\|} \operatorname{dif}\left(s_{x}, s_{y}\right)=\frac{1}{N(\mathbf{h})} \sum_{\operatorname{dis}\left(s_{x}, s_{y}\right)=\|\mathbf{h}\|} \frac{\sqrt{2}\left|s_{x}-s_{y}\right|}{2},
$$

where $N(\mathbf{h})$ denotes the number of paired samples whose separations are $\mathbf{h}$.

We could also consider the average of squared (perpendicular) distances as another measurement for the variation of paired samples separated by lag $\mathbf{h}$,

$$
\gamma(\mathbf{h})=\frac{1}{N(\mathbf{h})} \sum_{\operatorname{dis}\left(s_{x}, s_{y}\right)=\|\mathbf{h}\|}\left(\operatorname{dif}\left(s_{x}, s_{y}\right)\right)^{2}=\frac{1}{2 N(\mathbf{h})} \sum_{\operatorname{dis}\left(s_{x}, s_{y}\right)=\|\mathbf{h}\|}\left(s_{x}-s_{y}\right)^{2},
$$

which is the (semi) variogram or the moment of inertia for sample data.

The (semi) variogram of a function of random variables is defined as the half variance of difference between two continuous random variables separated by lag h,

$$
\gamma(\mathbf{h})=\frac{1}{2} \operatorname{Var}(V(x)-V(x+\mathbf{h})) .
$$

$\gamma(\mathbf{h})$ is a function only of the distance parameter $\mathbf{h}$, independent of the sample locations, (Noel , 1993). 


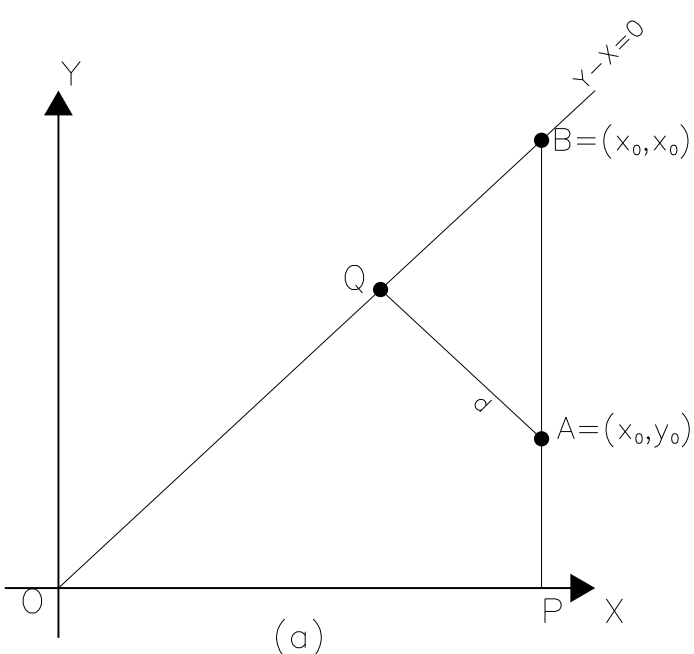

Figure 1: Distance index

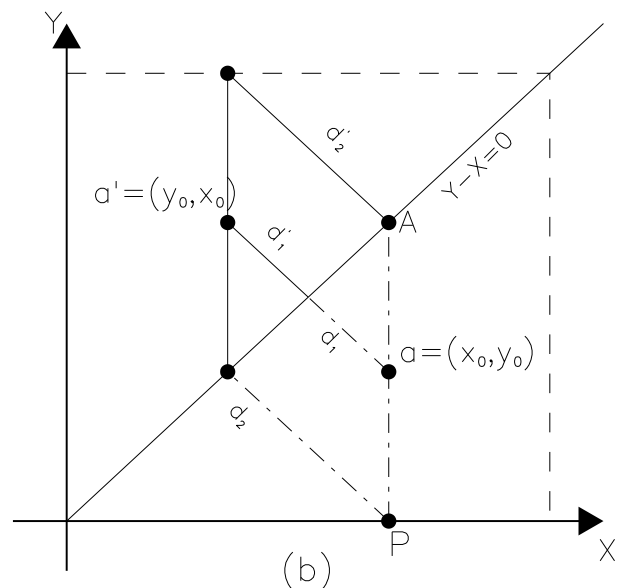

(b)

Since variance of a random variable is the expected values of squared differences from the mean of the random variable, the variogram can be expressed in term of expectation:

$$
2 \gamma(\mathbf{h})=\operatorname{Exp}\left(\{(V(x)-V(x+\mathbf{h}))-\operatorname{Exp}[V(x)-V(x+\mathbf{h})]\}^{2}\right) .
$$

This can be further simplified as

$$
2 \gamma(\mathbf{h})=\operatorname{Exp}\left((V(x)-V(x+\mathbf{h}))^{2}\right),
$$

assuming that the random function is stationary and the expectation between two points separated by $\mathbf{h}$ is $0, \operatorname{Exp}(V(x)-V(x+\mathbf{h}))=0$.

Finally, we see that the variogram of the continuous random function at separation $\mathbf{h}$ can be estimated or approximated by the sample variogram,

$$
\gamma(\mathbf{h})=\frac{1}{2} \operatorname{EXP}\left((V(x)-V(x+\mathbf{h}))^{2}\right) \approx \frac{1}{2 N(\mathbf{h})} \sum_{\operatorname{dis}\left(x_{o}, y_{o}\right)=\|\mathbf{h}\|}\left(x_{o}-y_{o}\right)^{2} .
$$

The statistical measurements of averaged orthogonal distances are useful as tests of spatial continuity hypotheses of underlying data sets. However, they may not be useful as measures of highly clustering data with significantly different stratum means. Measurement $\rho$ is directly pegged to the stratum mean, which makes it harder to be used for across-strata or across-variables comparisons, (smaller $\rho$ of a component or a variable does not necessarily indicate better spatial continuity than that of other components or variables). One possible 
remedy is to normalize the perpendicular ratio by using the orthogonal distance between paired samples with lag $\mathbf{h}$ over the maximum possible distance for the same paired samples. Let $a=\left(x_{o}, y_{o}\right)$ be a point in Figure $1(\mathrm{~b})$, then the ratio can be calculated as

$$
\delta_{1}=\frac{d_{1}}{d_{2}}=\frac{x_{o}-y_{o}}{x_{o}}, x_{o} \geq y_{o}
$$

and

$$
\delta_{2}=\frac{d_{1}^{\prime}}{d_{2}^{\prime}}=\frac{d_{1}}{d_{2}}=\frac{y_{o}-x_{o}}{y_{o}}, x_{o} \leq y_{o} .
$$

Combining the two expressions, yields:

$$
\delta=\frac{d_{1}}{d_{2}}=\frac{\left|x_{o}-y_{o}\right|}{\operatorname{Max}\left(x_{o}, y_{o}\right)}
$$

where $\operatorname{Max}\left(x_{o}, y_{o}\right)$ denotes the bigger element of $\left(x_{o}, y_{o}\right)$. The statistics based on the averaged ratio $\delta$ of all pairs of samples separated by lag $\mathbf{h}$ is as follows:

$$
\eta(\mathbf{h})=\frac{1}{N(\mathbf{h})} \sum_{\operatorname{dis}\left(s_{x}, s_{y}\right)=\|\mathbf{h}\|} \frac{\left|s_{x}-s_{y}\right|}{\operatorname{Max}\left(s_{x}, s_{y}\right)} .
$$

The new ratio $\eta$ gives us an index between 0 and 1 , where $\eta=\mathbf{0}$ indicates all points on the associated $h$-scatterplot are on the $\mathbf{y}=\mathbf{x}$ line. This is the perfect smoothness since any two samples with separation of lag $\mathbf{h}$ will have the same values. On the other hand, $\eta=1$ suggests the data set is extremely erratic since every pair of samples separated by lag $\mathbf{h}$ are maximally different. Both cases are extremely rare in practice, though. Finally, $0<\eta<1$ indicates the degree of closeness (or diffuseness) to the line of $\mathbf{y}=\mathbf{x}$ from all points on the $h$-scatterplot. Moreover, there is a strong geometric interpretation for the index $\eta$ : it indicates the area where about half of the points on the $h$-scatterplot are located. This is a sector started from the coordinate origin and bounded by the two rays on each side of the $\mathbf{y}=\mathbf{x}$ line. Geometrically and statistically speaking, the smaller the index $\eta$, the narrower the sector around the $\mathbf{y}=\mathbf{x}$ line, the smoother the data set represented by the $h$-scatterplot. On the other hand, the bigger the index, the wider the sector and the diffuser the data set.

It is easy to show that the equation for the line passing through the origin and $a=\left(x_{o}, y_{o}\right)$ in Figure $1(\mathrm{~b})$ is

$$
\frac{y}{x}=\frac{y_{o}}{x_{o}}=1-\frac{x_{o}-y_{o}}{x_{o}}=1-\delta_{1}, \quad x_{o} \geq y_{o}
$$

and

$$
\frac{x}{y}=\frac{x_{o}}{y_{o}}=1-\frac{y_{o}-x_{o}}{y_{o}}=1-\delta_{2}, \quad x_{o} \leq y_{o}
$$


Therefore, the sector associated with $\eta$ can be represented by the lines $\mathbf{y}=$ $(1-\eta) \mathbf{x}$ and $\mathbf{x}=(1-\eta) \mathbf{y}$. The slope of the line passing through the origin and the point $a$ can be expressed in term of $\delta$. Two points $P_{1}$ and $P_{2}$ on an $h$-scatterplot will be on the same line passing through the origin and the points $P_{1}$ and $P_{2}$ if and only if the ratios of $\delta$ associated with $P_{1}$ and $P_{2}$ are equal.

In summary, measurement $\eta$ can be interpreted in several ways: 1 ) it is an averaged ratio of the difference between pairs of samples separated by lag $\mathbf{h}$ over their maximum possible difference; 2 ) it is an averaged ratio of orthogonal distance from points to the $\mathbf{y}=\mathbf{x}$ line over the maximum possible orthogonal distance in the $h$-scatterplot; 3$)$ it is an averaged ratio of vertical distance from vertexes to the $\mathbf{y}=\mathbf{x}$ line over the maximum distance; 4) it is a sector bounded by two lines $\mathbf{y}=(1-\eta) \mathbf{x}$ and $\mathbf{x}=(1-\eta) \mathbf{y}$, where in average half of the points on the $h$-scatterplot are located.

\section{Analysis of Results}

Each of the new measurements was applied to all five parameters of stratified and non-stratified sample sets, respectively. In order to get some meaningful statistical summaries, we need to have a relatively large number of paired samples available for any given lag $\mathbf{h}$. The samples collected for the Lake Michigan Mass Balance Study were sparse. There are about 116 samples to cover 2318 cells of a $5 \mathrm{~km}$ by $5 \mathrm{~km}$ grid across the Lake Michigan. Very few samples, if any, will be separated exactly by $\|\mathbf{h}\|$ (cells). Therefore, the condition of paired samples separated exactly by lag $\mathbf{h}, \operatorname{dis}\left(s_{i}, s_{j}\right)=\|\mathbf{h}\|$, in calculating the spatial measurements needs to be relaxed to allow some tolerance $\mathbf{t},\|\mathbf{h}-\mathbf{t}\|<\operatorname{dis}\left(s_{i}, s_{j}\right)<\|\mathbf{h}+\mathbf{t}\|$. In addition, no spatial continuity in any specific direction has been observed in the sediment data set, northern direction with direction tolerance of $\pm 90^{\circ}$ is used in this study. The minimum lag is $\|\mathbf{h}\|=5$ cells with the distance and direction tolerance $\mathbf{t}=\left(0.5\right.$ cell, $\left.\pm 90^{\circ}\right)$ and the increment is 1 cell.

The snapshot of the comparisons for measurements $\rho$ and $\eta$ in Table 2, was produced for samples whose separations are $\mathbf{h}$ with tolerance $\mathbf{t}$. The differences between stratified and non-stratified sample sets for measurements $\rho$ and $\eta$ of all variables were investigated and summarized in the following tables and figures. From review of the results in Table 2, it is demonstrated that the spatial continuity, measured by the index $\rho$, of stratified sample data is much improved as compared to the spatial continuity measurement of unstratified sample sets. For example, the reading of $\rho$ with lag 5 for total phosphorus of the stratified sample sets is $0.1190,56 \%$ improvement over the reading of $\rho$ for the unstratified case, which is 0.2732 . The observations for other parameters in Table 2 are similar; the improvements of spatial continuity of stratified sample sets range from $26 \%$ (Biogenic Silica) to 56\% (total Phosphorus). 
Table 2: The comparisons of measurements $\rho$ and $\eta,\|\mathbf{h}\|=5$ cells

\begin{tabular}{lcccc}
\hline Parameter & $\rho$ (Stratified) & $\rho$ (All) & $\eta$ (Stratified) & $\eta$ (All) \\
\hline Total Phosphorus & 0.1190 & 0.2732 & 0.1163 & 0.2670 \\
NaOH Extr. Phosphorus & 0.0354 & 0.0514 & 0.1757 & 0.2549 \\
Organic Carbon & 4.7631 & 8.9902 & 0.1389 & 0.2621 \\
Total Organic Nitrogen & 0.6744 & 1.3143 & 0.1325 & 0.2582 \\
Biogenic Silica & 11.0571 & 14.8887 & 0.1478 & 0.1990 \\
\hline
\end{tabular}

Results and implications from examining the measurement $\eta$ are similar (Table 2). The measurements of $\eta$ on sediment parameters are also much lower when measured on stratified sample sets than on unstratified sample sets, which is consistent with the previous $\rho$ tests. We can also judge the spatial continuity of a data set from the associated scatterplots by the deviations of all vertices from the diagonal line. This information is illustrated in Figure 2, where the dashed lines are generated based on the measurement $\eta$, indicating how close (or how far), in average, the vertices are to the diagonal. The smaller the measurement $\eta$ is, the closer the vertices to the diagonal, the better spatial continuity of the data set. For example, the scatterplot for total phosphorus has $\eta$ of 0.1163 for the stratified sample set and 0.2670 for the unstratified sample set, a $56 \%$ improvement. In general, the improvements of $\eta$ range from 26\% (Biogenic Silica) to 56\% (total phosphorus). The comparisons are recorded in Figure 2. where red dashed lines indicate the improvements by data stratification and black dashed lines represent the measurements $\eta$ on the original samples (unstratified).

Two symbols, triangle and filled circle, representing vertices of scatterplots are used in the Figure 2, where circles are the vertices inside a stratum and triangles are the vertices of inter-strata. Recalling that each vertex in these scatterplots represents the values of a pair of samples within a certain distance, the perpendicular distance from the vertex to the diagonal line is used to measure the variation of the pair of the samples. The fact that most triangle symbols in the scatterplots of Figure 2 appear in upper-left or lower-right corners, the furthest locations away from the diagonal line in the first quadrant of the $\mathbf{x y}$-plane, clearly reveals that the pairs of nearby samples that belong to different components of the strata contribute the most for the larger reading of the measurement $\eta$ of unstratified sample sets. This phenomenon confirms and justifies the effectiveness of the usage of the measurement $\eta$ in evaluating proposed strata for a given distribution of data. The results demonstrated in Table 2 and Figure 2 are the measurements generated for lag $\|\mathbf{h}\|=5$ cells. The measurements generated for other lags are similar.

\section{Discussion}



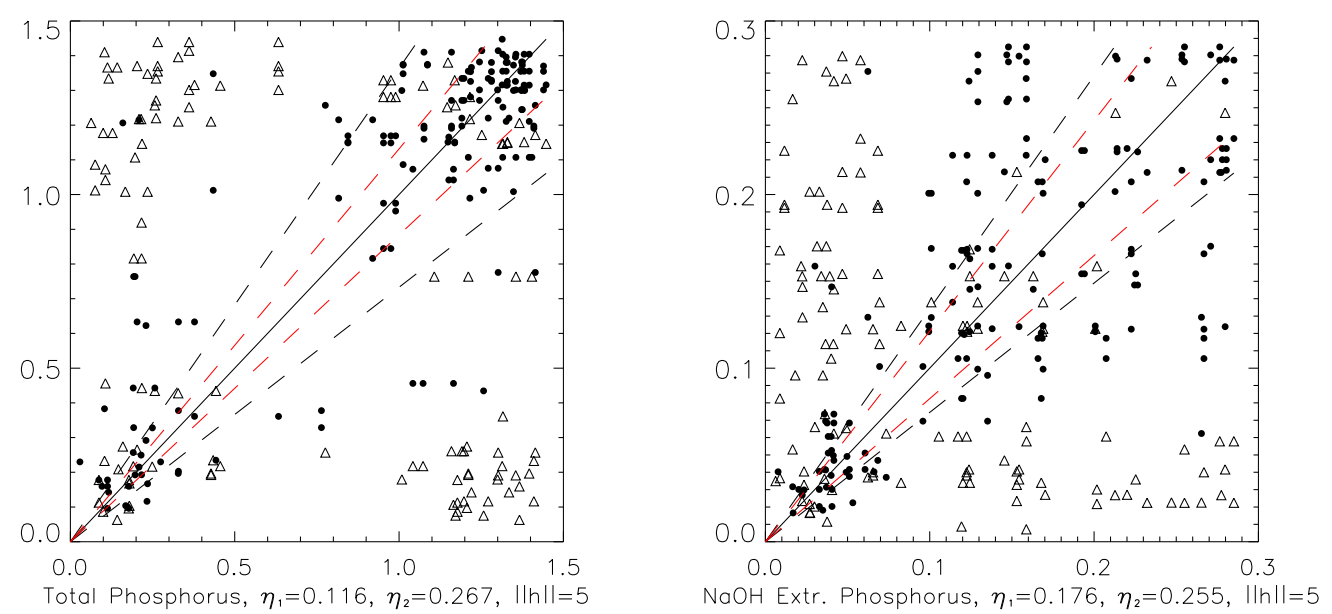

Figure 2: Comparisons of $\eta$ measurements, $\|\mathbf{h}\|=5$ cells

It has been recognized that the sediment data collected for the Lake Michigan Balance Study is not homogeneous across the lake. The distribution of sediment parameters correlates with the depth where samples are collected. This observation motivates us to classify the data into different categories based on the depth where various degrees of resuspension and settling of nutrients and carbon occur. Based on the frequency of the depth reached by waves, strata were estimated in section 1 as non-depositional, transitional, and depositional zones with depth range from 0 - 40 meters, 40 - 100 meters and 100 meters and deeper, respectively. As indicated by the previous section, all test results from spatial and descriptive statistics show that the variability of stratified data is much smaller than that of the data which are left unstratified.

The definition of strata may not be unique. Several different plausible explanations may produce different strata definitions. Though the idea of estimating strata based on applying the linear wave theory and examining sediment depth has been partially justified by test results from the last section, it is still desirable to ask whether the strata introduced in section 1 can be further improved in terms of fewer components, data continuity and homogeneity. An improved definition of strata should have: 1) fewer components, which makes more data available for use within an individual stratum; 2) larger difference of data attribution, which can be used to define strata, among individual components of the strata; and 3) maintenance of agreement with the understanding of the physics of the system (i.e., sediment concentrations of nutrients and carbon are correlated with depth). The application of the spatial statistical tools in conjunction with descriptive statistics suggests better stratification might exist, and the improvement for better strata configuration is possible. 
It can be observed from the concentration vs. depth plot (Figure 3) of total phosphorus samples that two groups of samples with different levels of measurements exist within the transitional zone, as shown in the left plot of Figure 3. The group of TP samples with higher concentrations is similar to that of the TP samples in the deeper depositional zone, while the other group of samples with lower concentrations is compatible with the samples at the shallow non-depositional zone where the depth is between 0 - 40 meters.
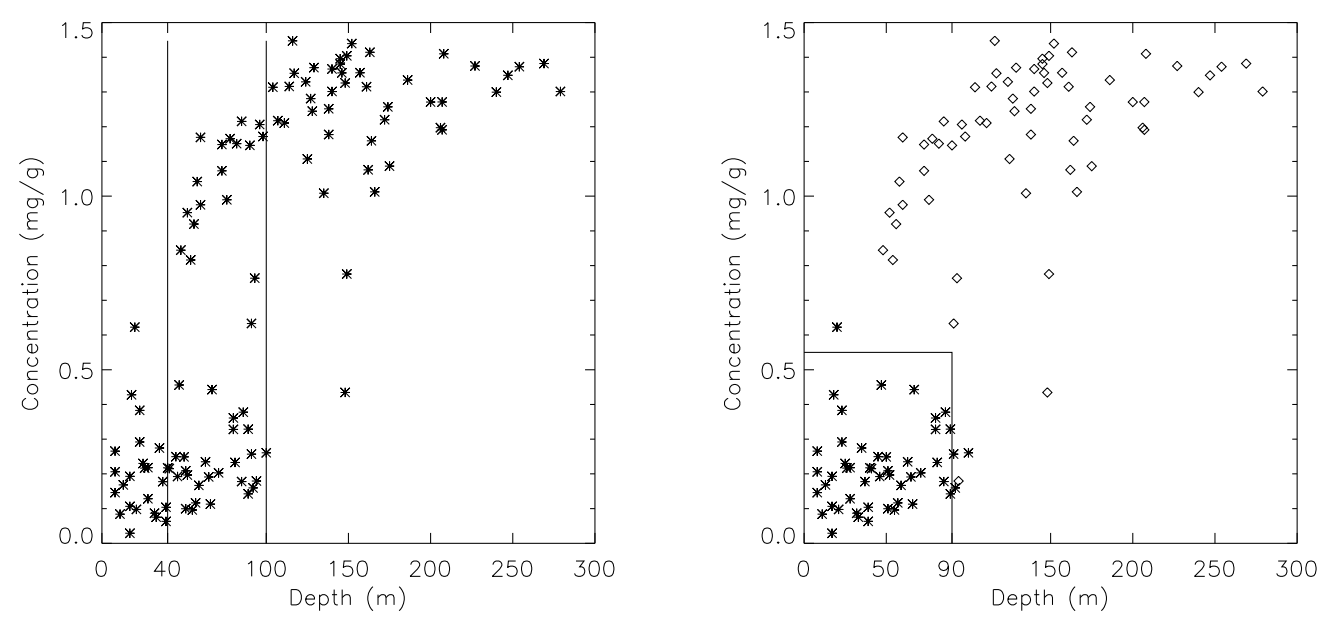

Figure 3: TP values vs. depth

This suggests that a transitional zone may not always be necessary in defining strata and the transitional zone could be merged with other strata. It turns out that these two groups indeed can be clearly separated geometrically. The group of higher measured samples is located at the offshore along the lower eastern shoreline of the lake which matches the area of plume described in the EEGLE report (Episodic Events - Great Lakes Experiment), which was initiated by the National Oceanic and Atmospheric Administration and the National Science Foundation to better understand near-shore to offshore mass exchange due to episodic events in the Great Lakes (EEGLE (2008)). This observation allows us to continue to define the strata based on the depth and topographic features. The new strata can be created as shown in the right half of Figure 3, where two symbols, diamonds and asterisks, represent the samples from different components, respectively.

The construction of the strata of two components could be partially justified by the EEGLE research program. Satellite imagery for Lake Michigan has captured a recurrent coastal plume in the southern portion of the lake (Eadie, Schwab, Leshkevich, Johengen, Assel, Holland, Hawley, Lansing, Lavrentyev, Miller, Morehead, Robbins, and VanHoof (1996)). In addition, measurements of 
currents made during the winter 2000 have suggested forced two-gyre circulation in the southern basin of the lake (Murthy, Rao, McCormick, Miller, and Saylor (2002)). Storm episodes have been suggested to generate significant offshore transport in this region. While the plume has been reported to appear along the entire southern coastline, it has also been reported to veer offshore along the eastern shore of the lake, which coincides with areas of largest measured long-term sediment accumulation (Schwab, Beletsky, and Lou (2000)). In considering these observations, it may be possible to create a delineation of strata for the Lake Michigan sediment bed that reflects a distribution which captures the movement of sediment from nearshore to offshore on the easternmost shoreline in the southern portion of the lake.

As an example of illustrating how two-zone stratification works for the sediment data set, a posting of a complete listing of locations and values of TP samples is shown in Figure 4(a). There are four kinds of samples based on the depths and locations. The samples with depths lower than 90 meters and the samples with depths deeper than or equal to 90 meters are represented by triangles and diamonds, respectively. In addition, a group of samples located along the southeast shore of the lake with high concentrations are represented by filled circles and a few samples whose depths are between 90 - 100 meters with low concentrations are represented by asterisks. All samples are plotted on the map of Lake Michigan, which is divided into two zones, the shallow zone and the deep zone, along the bathymetric line of 90 meters with some modification abovementioned. As illustrated in Figure 4(b), all samples collected at the depth less than 90 meters together with a few represented by asterisks are in the shallow zone, while the samples collected at the depth greater or equal to 90 meters together with the group of samples located along the southeast shore of the lake are in the deep zone of Lake Michigan. Figure 4(b) demonstrates that the lake is very effectively divided into two zones with values of samples of significant different magnitude. The values of samples from the deep zone are noticeably higher than that of the shallow zone. The patterns of other variables within the sediment data set are remarkably similar.

Descriptive statistics as well as spatial statistical summaries have been generated to compare different configurations of the strata containing three zones (non-depositional, transitional, and depositional) to a new definition of the strata containing two zones, the shallow zone and the deep zone. Means and standard deviations of all parameters of different zones from both definitions of strata are given in Table 3, where the non-depositional zone and the depositional zone will be directly compared to the shallow zone and the deep zone, respectively. The summary from Table 3 reveals that for all variables, the means of the shallow zone are a little higher than the means of the non-depositional zone, while the 

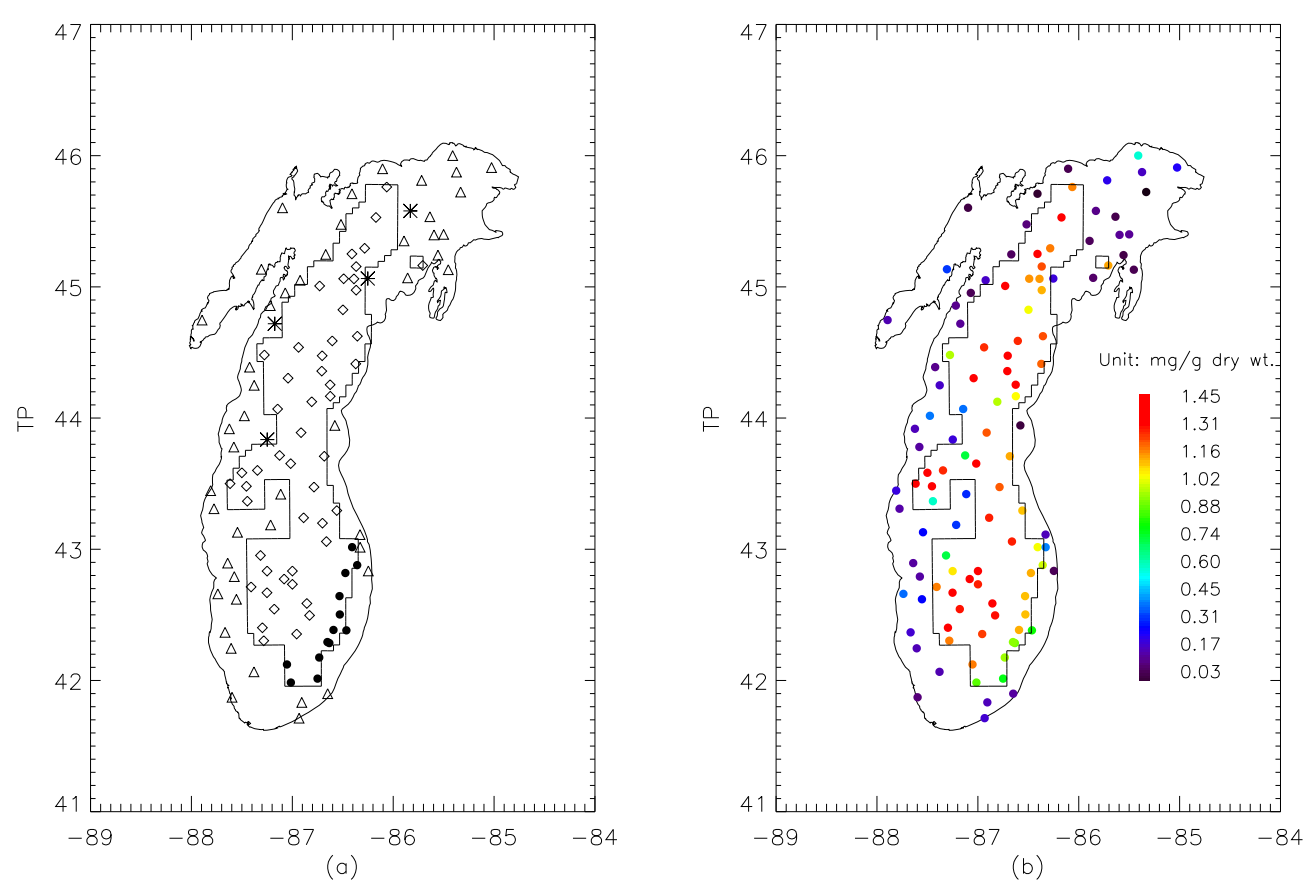

Figure 4: The distribution of TP samples in the strata of two zones

means of deep zone are a little lower than that of the depositional zone. This reflects the fact that the majority of additional samples in the new shallow zone are from the old transitional zone with values higher than the mean of the nondepositional zone samples (Figure 3 ). Similarly the majority of additional samples in the new deep zone are also from the old transitional zone but with values lower than the mean of the depositional zone samples. On the other hand, the standard deviations of variables from the shallow zone are all smaller than that of the nondepositional zone, while the standard deviations of variables from the deep zone are a little higher than that of the depositional zone. It is not surprising to find that the variances within the transitional zone for all variables except Biogenic Silica are as large as those of the depositional zone and the deep zone where sample values are much higher. This reflects the fact that samples collected from the transitional zone vary greatly and are not evenly distributed. The transitional could be eliminated when strata are to be defined. Most importantly, all variables (except Biogenic Silica) of two-zone strata show less variation than that of three-zone strata and both noticeably improve in terms of variation over that of unstratified samples as indicated by the standard deviations from Table 3. This finding is significant. In general for the same sample set, the more components a strata has the less variation the strata since the smaller stratum configuration usually reduces the variability, and data inside the smaller stratum 
is more homogeneous compared to the larger stratum. The reverse is true when comparing the variances of two-zone strata with bigger strata components with that of three-zone strata with smaller components, which strongly indicates that data within two-zone strata are more smooth and homogeneous.

The summaries of the application of spatial statistics, both the measurements of $\rho$ and $\eta$ of lag $\|\mathbf{h}\|=5$ of all variables, are given in Table 4 . The most important discovery from Table 4, in terms of data continuity measured by $\rho$ and $\eta$, is that samples sets of the new strata of two zones are better than that of the strata of three zones and both are considerable improvements over sample sets left unstratified. Furthermore, this observation is also true for all lags of 5 to 65 cells of $5 \mathrm{~km}$ grid cells as suggested by Figure 5, where black, green, and red lines are representing the $\rho$ and $\eta$ measurements of all samples without stratification, sample strata of three zones, and sample strata of two zones respectively. Measurements $\rho(\mathbf{h})$ and $\eta(\mathbf{h})$ for samples of strata are calculated by using formulas similar to (4) and (13) of Section 2, except that only samples in the same zone of strata can be used to calculate the variation of paired samples separated by lag $\mathbf{h}$. The prevailing patterns illustrated in Figure 5 are that the black lines are significantly higher than both green and red lines, and the green lines are higher than the red ones, which provides strong evidence to support a data stratification approach for better data applications.

Combining descriptive statistics and spatial measurements can provide a better description of the distribution patterns of the sediment data under concern. Both descriptive and spatial statistics justify the definitions of strata of three zones or strata of two zones for the sediment samples, and both of these definitions are significantly better than the original unstratified samples in terms of spatial continuity as well as data variation and data distribution. All statistical analysis of the current data, both descriptive statistics and spatial statistics, indicate that data application will benefit from data stratification if the three-zone strata (non-depositional, transitional, and depositional zones) can be reformed as a new two-zone strata (shallow and deep zones). In addition, the new twozone strata formation is much better in terms of significant difference between the two components. The most important criterion in defining strata is to find the significant attributions of data which can be used to group data. While the differences (of means) between two adjacent zones of three-zone strata is about $200 \%-300 \%$, the differences between the shallow and deep zones of the two-zone strata is much higher, ranging from $600 \%-1100 \%$. This alone suggests the new strata with two zones result in a much greater deviation between zones. Further, when some applications such as data interpolation need to be applied to each individual zone of the strata, there will be more samples available when the number of zones is reduced. Using the collected field data set from the Lake Michigan 
Table 3: Statistical comparison of 2 different definitions of strata

\begin{tabular}{|c|c|c|c|c|c|c|}
\hline \multirow{3}{*}{$\begin{array}{l}\text { Zone Conf. } \\
\text { Measurements }\end{array}$} & \multicolumn{4}{|c|}{ Total Phosphorus (Unit: $\mathrm{mg} / \mathrm{g}$ dry wt.) } & \multirow{2}{*}{\multicolumn{2}{|c|}{ Std. Div. }} \\
\hline & \multicolumn{2}{|c|}{ Data Range } & \multicolumn{2}{|c|}{ Mean } & & \\
\hline & 3-Zones & 2-Zones & 3-Zones & 2-Zones & 3-Zones & 2-Zones \\
\hline Non-Dep. Zone & $0.029-0.623$ & $0.029-0.623$ & 0.201 & 0.217 & 0.134 & 0.116 \\
\hline Trans. Zone & $0.096-1.215$ & - & 0.547 & - & 0.413 & - \\
\hline Dep. Zone & $0.435-1.447$ & $0.435-1.447$ & 1.255 & 1.188 & 0.182 & 0.207 \\
\hline Stratified & $0.029-1.447$ & $0.029-1.447$ & 0.753 & 0.753 & 0.290 & 0.172 \\
\hline All Samples & $0.029-1.447$ & $0.029-1.447$ & 0.753 & 0.753 & 0.514 & 0.514 \\
\hline
\end{tabular}

Sodium Hydroxide Extractable Phosphorus (Unit: mg/g dry wt.)

Zone Conf.

Data Range

Mean

\begin{tabular}{lcccccc}
\cline { 5 - 6 } Measurements & 3-Zones & 2-Zones & 3-Zones & 2-Zones & 3-Zones & 2-Zones \\
\hline Non-Dep. Zone & $0.007-0.147$ & $0.006-0.147$ & 0.033 & 0.036 & 0.029 & 0.023 \\
Trans. Zone & $0.006-0.247$ & - & 0.076 & - & 0.055 & - \\
Dep. Zone & $0.062-0.285$ & $0.061-0.285$ & 0.193 & 0.175 & 0.059 & 0.063 \\
Stratified & $0.006-0.285$ & $0.006-0.285$ & 0.113 & 0.113 & 0.053 & 0.049 \\
All Samples & $0.006-0.285$ & $0.006-0.285$ & 0.113 & 0.113 & 0.085 & 0.085 \\
\hline
\end{tabular}

\begin{tabular}{|c|c|c|c|c|c|c|}
\hline \multirow{3}{*}{$\begin{array}{l}\text { Zone Conf. } \\
\text { Measurements }\end{array}$} & \multicolumn{4}{|c|}{ Organic Carbon (Unit: mg/g dry wt.) } & \multirow{2}{*}{\multicolumn{2}{|c|}{ Std. Div. }} \\
\hline & \multicolumn{2}{|c|}{ Data Range } & \multicolumn{2}{|c|}{ Mean } & & \\
\hline & 3-Zones & 2-Zones & 3-Zones & 2-Zones & 3-Zones & 2-Zones \\
\hline Non-Dep. Zone & $0.400-21.20$ & $0.400-21.20$ & 3.436 & 3.508 & 4.396 & 3.465 \\
\hline Trans. Zone & $0.500-48.51$ & $\longrightarrow$ & 12.763 & - & 12.532 & - \\
\hline Dep. Zone & $10.28-47.875$ & $10.28-48.51$ & 33.68 & 31.347 & 6.802 & 8.223 \\
\hline Stratified & $0.400-48.51$ & $0.400-48.51$ & 18.868 & 18.868 & 9.187 & 6.534 \\
\hline All Samples & $0.400-48.51$ & $0.400-48.51$ & 18.868 & 18.868 & 15.353 & 15.353 \\
\hline
\end{tabular}

\begin{tabular}{|c|c|c|c|c|c|c|}
\hline \multirow{3}{*}{$\begin{array}{l}\text { Zone Conf. } \\
\text { Measurements }\end{array}$} & \multicolumn{6}{|c|}{ Total Organic Nitrogen (Unit: mg/g dry wt.) } \\
\hline & \multicolumn{2}{|c|}{ Data Range } & \multicolumn{2}{|c|}{ Mean } & \multicolumn{2}{|c|}{ Std. Div. } \\
\hline & 3-Zones & 2-Zones & 3-Zones & 2-Zones & 3-Zones & 2-Zones \\
\hline Non-Dep. Zone & $0.100-3.200$ & $0.100-3.200$ & 0.588 & 0.593 & 0.683 & 0.517 \\
\hline Trans. Zone & $0.100-7.200$ & - & 1.918 & - & 1.775 & - \\
\hline Dep. Zone & $2.400-7.050$ & $2.400-7.200$ & 5.041 & 4.704 & 0.964 & 1.113 \\
\hline Stratified & $0.100-7.200$ & $0.100-7.200$ & 2.812 & 2.812 & 1.308 & 0.890 \\
\hline All Samples & $0.100-7.200$ & $0.100-7.200$ & 2.812 & 2.812 & 2.241 & 2.241 \\
\hline \multicolumn{7}{|c|}{ Biogenic Silica (Unit: mg/g dry wt.) } \\
\hline Zone Conf. & \multicolumn{2}{|c|}{ Data Range } & \multicolumn{2}{|c|}{ Mean } & \multicolumn{2}{|c|}{ Std. Div. } \\
\hline Measurements & 3-Zones & 2-Zones & 3-Zones & 2-Zones & 3-Zones & 2-Zones \\
\hline Non-Dep. Zone & $0.313-34.11$ & $0.182-34.11$ & 4.520 & 4.526 & 7.398 & 5.444 \\
\hline Trans. Zone & $0.182-40.44$ & - & 10.87 & - & 9.975 & - \\
\hline Dep. Zone & $17.62-105.8$ & $4.196-105.8$ & 51.43 & 42.418 & 26.338 & 26.733 \\
\hline Stratified & $0.182-105.8$ & $0.182-105.8$ & 25.32 & 25.316 & 17.927 & 20.136 \\
\hline All Samples & $0.182-105.8$ & $0.182-105.8$ & 25.32 & 25.316 & 27.589 & 27.589 \\
\hline
\end{tabular}


Table 4: The Comparisons of Measurement $\rho$ and $\eta,\|\mathbf{h}\|=5$

\begin{tabular}{|c|c|c|c|c|}
\hline \multirow{2}{*}{$\begin{array}{l}\text { Zone Conf. } \\
\text { Measurements }\end{array}$} & \multicolumn{2}{|c|}{ RHO Measurement } & \multicolumn{2}{|c|}{ ETA Measurement } \\
\hline & 3-Zones & 2-Zones & 3-Zones & 2-Zones \\
\hline Stratified & 0.1190 & 0.1126 & 0.1163 & 0.1100 \\
\hline Unstratified & 0.2732 & 0.2732 & 0.2670 & 0.2670 \\
\hline \multicolumn{5}{|c|}{ Sodium Hydroxide Extractable Phosphorus } \\
\hline Zone Conf. & \multicolumn{2}{|c|}{ RHO Measurement } & \multicolumn{2}{|c|}{ ETA Measurement } \\
\hline Measurements & 3-Zones & 2-Zones & 3-Zones & 2-Zones \\
\hline Stratified & 0.0354 & 0.0341 & 0.1757 & 0.1692 \\
\hline Unstratified & 0.0514 & 0.0514 & 0.2549 & 0.2549 \\
\hline \multicolumn{5}{|c|}{ Organic Carbon } \\
\hline Zone Conf. & \multicolumn{2}{|c|}{ RHO Measurement } & \multicolumn{2}{|c|}{ ETA Measurement } \\
\hline Measurements & 3-Zones & 2-Zones & 3-Zones & 2-Zones \\
\hline Stratified & 4.7631 & 4.2031 & 0.1389 & 0.1225 \\
\hline Unstratified & 8.9902 & 8.9902 & 0.2621 & 0.2621 \\
\hline \multicolumn{5}{|c|}{ Total Organic Nitrogen } \\
\hline Measurements & 3-Zones & 2-Zones & 3-Zones & 2-Zones \\
\hline Stratified & 0.6744 & 0.5504 & 0.1325 & 0.1081 \\
\hline Unstratified & 1.3143 & 1.3143 & 0.2582 & 0.2582 \\
\hline \multicolumn{5}{|c|}{ Biogenic Silica } \\
\hline Zone Conf. & \multicolumn{2}{|c|}{ RHO Measurement } & \multicolumn{2}{|c|}{ ETA Measurement } \\
\hline Measurements & 3-Zones & 2-Zones & 3-Zones & 2-Zones \\
\hline Stratified & 11,0571 & 10.2060 & 0.1478 & 0.1364 \\
\hline Unstratified & 14.8887 & 14.8887 & 0.1990 & 0.1990 \\
\hline
\end{tabular}

Mass Balance Study, this data analysis suggests that the transitional zone of the three-zone strata should be merged into other zones to establish the new two-zone strata.

All analyses done in the current exercise were based on the sediment samples collected for the Lake Michigan Mass Balance Study. There are 116 samples across the entire Lake Michigan, with surface area of 60,000 square km, resulting in a density of one sample over 500 square $\mathrm{km}$, or one sample per 20 cells of a $5 \mathrm{~km}$ by $5 \mathrm{~km}$ grid. The lack of sufficient sampling coverage limited our ability to perform a more thorough analysis and required the use of a larger lag tolerance 

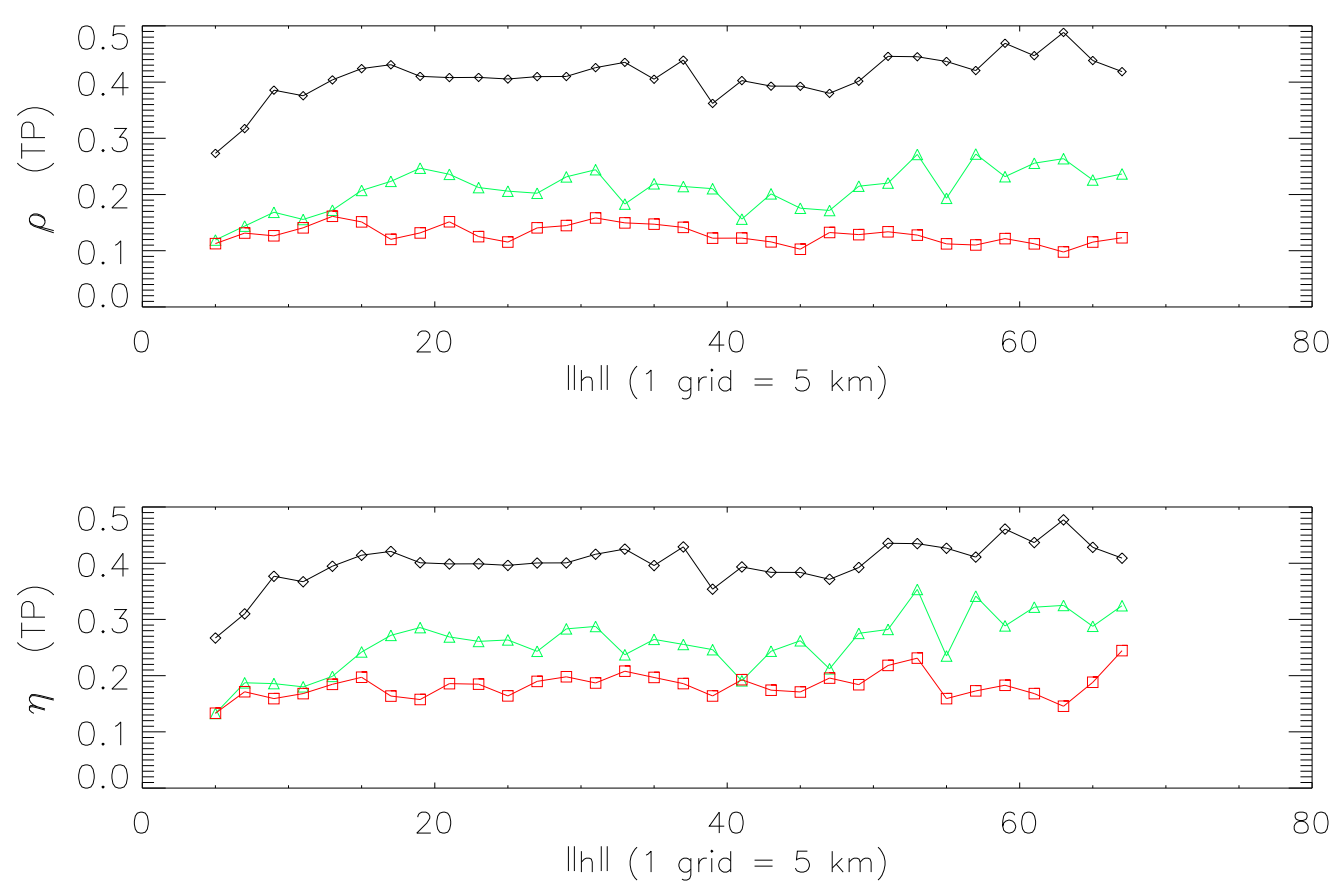

Figure 5: The measurements of $\rho$ and $\eta$ of three configurations of strata

when spatial variation was calculated. An enhanced sampling design with a larger number of sample sites and a better representativeness would allow for a more rigorous analysis and a better understanding of the distribution of data sets for a given variable, and in turn a better definition of stratification.

\section{Conclusion}

The National Oceanic and Atmospheric Administration (N.O.A.A.), National Data Buoy Center (N.D.B.C.) maintains Buoys in each of the Great Lakes that monitor for wave heights in addition to several other parameters that have been utilized in analysis of Great Lakes wave patterns (Plattner, Mason, Leshkevich, Schwab, and Rutherford (2006)). In the present study, multiple quantitative evaluators were developed that demonstrated the employment of a series of quantitative scores to evaluate the spatial distribution of environmental measurements. Specifically, phosphorus, nitrogen, silica, and carbon distributions in the sediment were investigated for a large freshwater lake, Lake Michigan. Observational data (sediment concentrations of nutrients and carbon) were analyzed with respect to the underlying physics governing the system under observation (wave action), and a novel quantitative approach was utilized to justify a stratification of the observational data in agreement with the physics of the system driven by linear 
wave theory. This study has important implications for concurrent research that focuses on examining eutrophication processes in Lake Michigan (and each of the Great Lakes) for which nitrogen, silica, and phosphorus cycle from the sediment through the lower food web of the lake.

Water quality models have been formulated and applied to the Great Lakes (and Lake Michigan in particular) to investigate the fate and transport of nutrients, to forecast phosphorus and nitrogen concentrations, and to manage eutrophication (Canale, Depalma, and Vogel (1975); Chapra, and Sonzogni (1979); Rodgers, and Salisbury (1981); Scavia (1988); Lesht, Fontaine, and Dolan (1991); Chen, Rubao, Schwab, Beletsky, Fahnenstiel, Jiang, Johengen, Vanderploeg, Eadie, Budd, Bundy, Gardner, Cotner, and Lavrentyev (2002)). Within these models, the exchange of nutrients between the sediment concentrations and overlying water column is a critical component, particularly in considering near shore areas, whereby the overlying water column allows for phytoplankton to exist at all depths. Credible site specific exchanges between the sediment and water column within a water quality model depend upon the ability to accurately predict the distribution of nutrients across the sediment bed of the water body.

In addition. the current study has important applications in understanding contaminant fate and transport processes within Lake Michigan (which are not independent on eutrophication processes), including bioaccumulation studies for hydrophobic chemicals such as polychlorinated hydrocarbons (PCBs) that are distributed in the sediment, and which are continually reintroduced to the water column via resuspension processes driven by wave dynamics. Several PCB congeners exhibit a large hydrophobicity, and therefore the distribution of carbon in the sediment bed of Lake Michigan provides an insight as how PCB congeners will distribute themselves. Several water quality models are carbon based models in which the kinetics of carbon within these models is the driving mechanism for the dynamics of hydrophobic toxic chemicals. As an example, the Lake Michigan Mass Balance Study utilized biota boxes to estimate PCB congener interactions between sediment and the overlying water column and resulting concentrations to the food web of the lake at these locations. These boxes were large enough to encompass multiple strata as predicted using linear wave theory and the quantitative scores developed in the present study. Therefore, employment of a stratification may be necessary to produce an accurate exposure concentration for PCB congeners to the food web at these locations. Further, toxic chemicals that bind to carbon such as PCBs exist in all the Great Lakes as well as other water bodies. Accurately estimating sediment concentration of toxic chemicals through the use of appropriate sampling designs that can account for spatial variability allows for an accurate estimate of site specific exposure concentrations. Methods have been developed to translate site specific exposures to toxic chemicals into population 
level effects, given that exposures can be linked to vital rates (Miller, Jensen, Villeneuve, Kahl, Makynen, and Durhan (2007); Miller, and Ankley (2004); Brown, Riddle, Cunningham, Kedwards, Shillabeer, and Hutchinson (2003)). Therefore, accurately describing the distribution of sediment concentrations of toxic chemicals will contribute to more precise ecological risk assessments corresponding to specific contaminated sites within a water body.

\section{Acknowledgements}

We thank David J. Schwab, Lawrence P. Burkhard and Ronald Rossmann for providing a valuable review of this manuscript. The authors also wish to acknowledge the Great Lakes National Program Office for their efforts in the Lake Michigan Mass Balance Study, as well as many cooperators during the study. This paper has been reviewed according to ORD guidelines, but the statements made do not represent views of the USEPA, nor does mention of trade names indicate endorsement by the federal government.

\section{References}

Brown, A. R., Riddle, A. M., Cunningham, N. L., Kedwards, T. J., Shillabeer, N., and Hutchinson, T. H. (2003). Predicting the effects of endocrine disrupting chemicals on fish populations. Human and Ecological Risk Assessment 9(3), 761-788.

Burkhard, L. P. (2003). Factors influencing the design of BAF and BSAF field studies. Environ. Toxicol. Chem. 22, 351-360.

Canale, R. P., Depalma, L. M., and Vogel., A. H. (1975). A plankton based food web model for Lake Michigan. Chapter 2 in: Modeling Biochemical Processes in Aquatic Ecosystems. Sea Grant Program, Ann Arbor, Michigan, 33-74.

Chapra, S. C. and Sonzogni., W. C. (1979). Great lakes total phosphorus budget for the mid 1970s. Journal of the Water Pollution Control Federation 51(10), 2524-2533.

Chen, C., Rubao, J., Schwab, D. J., Beletsky, D., Fahnenstiel, G. L., Jiang, M., Johengen, T. H., Vanderploeg, H., Eadie, B., Budd, J. W., Bundy, M. H., Gardner, W., Cotner, J., and Lavrentyev., P. J. (2002). A model study of the coupled biological and physical dynamics in Lake Michigan. Ecological Modelling 152, 145-168.

Cook, P. M., Robbins, J. A., Endicott, D. D., Lodge, K. B., Guiney, P. D., Walker, M. K., Zabel, E. W., and Peterson, R. E. (2003). Effects of aryl hydrocarbon receptormediated early life stage toxicity on lake trout populations in Lake Ontario during the 20th century. Environ. Sci. Technol. 37, 3864-3877.

Craik, A. D. D. (2004). The origins of water wave theory. Annual Review of Fluid Mechanics 36, 1-28.

Cressie, N. (1993). Statistics for Spatial Data. John Wiley \& Sons, New York. 
Dean, R. G. and Dalrymple, R. A. (1991). Water wave mechanics for engineers and scientists. Advanced Series on Ocean Engineering 2. World Scientific, Singapore.

Eadie, B. J., D. J. Schwab, G. L. Leshkevich, T. H. Johengen, R. A. Assel, R. E. Holland, N. Hawley, M. B. Lansing, P. Lavrentyev, G. S. Miller, N. R. Morehead, J. A. Robbins, and VanHoof, P. L. (1996). Anatomy of a recurrent episodic event: a winter-spring plume in southern Lake Michigan, EOS. Transactions of the American Geophysical Union 77, 337-338.

EEGLE. (2008). EEGLE: Episodic Events Great Lakes Experiment. http://www.glerl. noaa.gov/eegle/. Cited 04 June 2008.

Hook, T. A., Rutherford, E. S., Brines, S. J., Geddes, C. A., Mason, D. M., Schwab, D. J. and Fleischer, G. W. (2004). Landscape scale measures of steelhead (Oncorhynchus mykiss) Bioenergetic growth rate potential in Lake Michigan and comparison with angler catch rates. J. Great Lakes Res. 30(4), 545-556.

Isaaks, E. and Srivastava, M. (1989). Applied Geostatistics. Oxford University, Oxford.

Lesht, B. M., Fontaine, T. D., and Dolan., D. M. (1991). Great Lakes total phosphorus model: post-audit and regionalized sensitivity analysis. J. Great Lakes Res. 17(1), 3-17.

Madenjian, C. P., DeSorcie, T. J., Stedman, R. M., Brown, E. H. Jr., Eck, G. W., Schmidt, L. J., Hesselberg, R. J., Chernyak, S. M., and Passino-Reader, D. R. (1999). Spatial Patterns in PCB Concentrations of Lake Michigan Lake Trout. J. Great Lakes Res. 25(1), 149-159.

Madenjian, C. P., Elliott, R. F., Schmidt, L. J., Desorcie, T. J., Hesselberg, R. J., Quital, R. T., Begnoche, L. J., Bouchard, P. M., and Holey, M. E. (1998). Net trophic transfer efficiency of PCBs to Lake Michigan Coho Salmon from their prey. Environ. Sci. Technol. 32, 3063-3067.

Matheron, G. (1962). Treatise on Applied Geostatistics. Part I, Paries, France.

Miller, D. H. and Ankley, G. T. (2004). Modeling impacts on populations: fathead minnow (Pimephales promelas) exposure to the endocrine disruptor 17B-Trenbolone as a case study. Ecotoxicol. Environ. Saf. 59, 1-9.

Miller, D. H., Jensen, K. M., Villeneuve, D. L., Kahl, M. D., Makynen, E. A., Durhan, E. J., et al. (2007). Linkage of biochemical responses to population-level effects: a case study with vitellogenin in the fathead minnow (Pimephales promelas). Environ. Toxicol. Chem. 26, 521-527.

Miller, D. H., Xia, X., Huang, W. and Rossmann, R. (2010). Distribution of sediment measurements in Lake Michigan as a case study. Implications for Estimating Sediment-Water Interactions in Eutrophication and Bioaccumulation Models. Preprint.

Mladenoff, D. J., Sickley, T. A., Haight, R. G., and Wydeven, A. P., (1995). A regional landscape analysis and prediction of favorable gray wolf habitat in the northern Great Lakes region. Conservation Biology 9, 279-294. 
Mladenoff, D. J., Haight, R. G., Sickley, T. A., and Wydeven, A. P., (1997). Causes and implications of species restoration in altered ecosystems. Bioscience 47(1), 21-31.

Moore, D. W., Baudo, R., Conder, J. M., Landrum, P. F., McFarland, V. A., Meador, J. P., Millward, R. N., Shine, J. P., and Word., J. Q. (2005). Bioaccumulation in the assessment of sediment quality: uncertainty and potential application. In: Use of sediment quality guidelines and related tools for the assessment of contaminated sediments, Wenning, R. J., Batley, G. E., Ingersoll, C. G., and D. W. Moore eds. Society of Environmental Toxicology and Chemistry.

Murthy, C.R., Rao, Y.R., McCormick, M.J., Miller, G.S. and Saylor, J. H. (2002). Coastal exchange characteristics during unstratified season in southern Lake Michigan. Verh. Internat. Verein. Limnol. 28, 229-302.

Plattner, S., Mason, D. M., Leshkevich, G. A., Schwab, D. J., and Rutherford., E. S. (2006). Classifying and forecasting coastal upwellings in Lake Michigan using satellite derived temperature images and buoy data. J. Great Lakes Res. 32, 63-76.

Scavia, D. et al. (1988). Dynamics of Lake Michigan plankton: a model evaluation of nutrient loading, competition, and predation. Canadian Journal of Fisheries and Aquatic Sciences 45, 165-177.

Schwab, D. J., Beletsky, D. and Lou, J. (2000). The 1998 Coastal Turbidity Plume in Lake Michigan. Est. Coast. Shelf Sci. 50, 4958, 2000.

Rao, Y. R., Murthy, C. R., McCormick, M. J., Miller, G. S., and Saylor, J. H. (2002). Observations of circulation and coastal exchange characteristics in southern Lake Michigan during 2000 winter season. Geophys. Res. Letters 29(13), 9-1 to 9-4.

Rodgers, P. W. and Salisbury., D. (1981). Water quality modeling of Lake Michigan, and consideration of the anomalous ice cover for 1976-1977. J. Great Lakes Res. $7(4), 467-480$.

Rossmann, R. and Seibel, E. (1977). Surficial sediment redistribution by wave energy: element-grain size relationship. J. Great Lakes Res. 3(3-4), 258-262.

U.S. Environmental Protection Agency. (1997). Lake Michigan Mass Balance Study (LMMB) Methods Compendium, Volume 1: Sample Collection Techniques. U.S. Environmental Protection Agency, Great Lakes National Program Office, Chicago, Illinois. EPA/905/R-97/012a.

Received August 24, 2009; accepted January 18, 2010. 
Xiangsheng Xia

EPA Large Lakes Research Station

CSC Corporation

9311 Groh, Grosse Ile, MI 48138, USA

Email: xia.xiangsheng@epa.gov

David H. Miller

U.S. EPA, ORD, NHEERL

Mid-Continent Ecology Division

Large Lakes Research Station

9311 Groh, Grosse Ile, MI 48138, USA

Email: miller.davidh@epa.gov 